\title{
Endoscopic Stenting for Malignant Hilar Biliary Obstruction: After You Double Down, Are You In or Out?
}

\author{
Nanlong Liu ${ }^{1}$. Dennis Yang ${ }^{2} \cdot$ Peter V. Draganov ${ }^{2}$ \\ Published online: 15 April 2020 \\ (c) Springer Science+Business Media, LLC, part of Springer Nature 2020
}

Due to the presence of advanced disease, the majority of patients with malignant biliary hilar obstruction are not candidates for curative surgical resection, leaving palliative surgical, percutaneous, or endoscopic biliary drainage as the most important tool used to improve patient quality-of-life [1]. Despite the lack of high-quality comparative data, placement of stents via endoscopic retrograde cholangiopancreatography (ERCP) has become the preferred approach [2]. Placement of metal compared with plastic stents provides longer patency and is more cost-effective [3]. A goal of at least $50 \%$ drainage of the obstructed liver has been associated with improved clinical outcomes and survival [4]. Recent studies suggest that bilateral stenting may be associated with fewer interventions, more durable stent patency, and better biliary drainage [5]. If bilateral metal stent placement is planned, this can be achieved by placing the two stents side-by-side (SBS) into the two opposite lobes of the liver. Alternatively, after placing the first stent into one liver lobe, the second stent can be inserted through the first into the opposing lobe in a $\mathbf{Y}$ configuration termed the stent-instent (SIS) technique (Figs. 1, 2).

Though the SBS and SIS approaches are routinely used in everyday practice, only a few studies have attempted to compare the two techniques. In one retrospective study, Naitoh et al. found both methods had similar success rates, but that SBS had longer stent patency but a higher complication rate [6]. In a separate retrospective study, however, there was no

Nanlong Liu

nathanliu9@gmail.com

1 Division of Gastroenterology, Hepatology \& Nutrition, Department of Medicine, University of Louisville, 550 South Jackson St., 3rd Floor, Ste. A3L15, Louisville, KY 40202, USA

2 Division of Gastroenterology, Hepatology \& Nutrition, Department of Medicine, Department of Medicine in the College of Medicine, University of Florida, Gainesville, FL, USA difference in success rates, complication rates, or patency time between the methods [7].

In this issue of Digestive Diseases and Sciences, Ishigaki et al. retrospectively studied the outcomes of 64 patients with malignant hilar biliary obstruction with data obtained from four different hospitals over the course of 5.5 years. There were 24 patients in the SBS group and 40 in the SIS group. For SBS, the 8-mm uncovered Wallflex ${ }^{\mathrm{TM}}$ (Boston Scientific Corporation, Marlborough, MA, USA) biliary stents were used and placed across the papilla [8]. For SIS, the 10-mm uncovered Niti-S ${ }^{\text {TM }}$ large cell D-type stents (LCD; Taewoong Corp., Gimpo, Korea) were used and placed above the papilla. Biliary sphincterotomy was performed on all SBS patients, but was performed at the discretion of the endoscopist in the SIS group. There was no statistical difference in choice of stent method regarding clinical characteristics including age, cause of obstruction, stage of the malignancy, and Bismuth classification. The study was also performed chronologically with SIS method used in the initial study period and the SBS method in the later time period.

In their study, both methods carried a high technical success $(96 \%$ SBS vs $100 \%$ SIS) with no statistical difference between the rates of recurrent biliary obstruction (RBO; 43-48\%), numbers of ERCPs in the follow-up period (two), and average survival rate (381 days). Notably, post-procedure pancreatitis was observed in seven patients, exclusively in the SBS group. A possible explanation could be that the SBS stents placed across the papilla may exert higher pressure on the pancreatic duct orifice; albeit a prior study by Shin et al. did not find a higher complication rate with transpapillary placement in comparison with supra-papillary placement [9]. Another potential explanation offered by the authors is the higher number of trainees involved with SBS stenting as compared to SIS. Nonetheless, whether trainee involvement could have accounted for this difference remains unclear, as a recent study by Voiosu and colleagues 


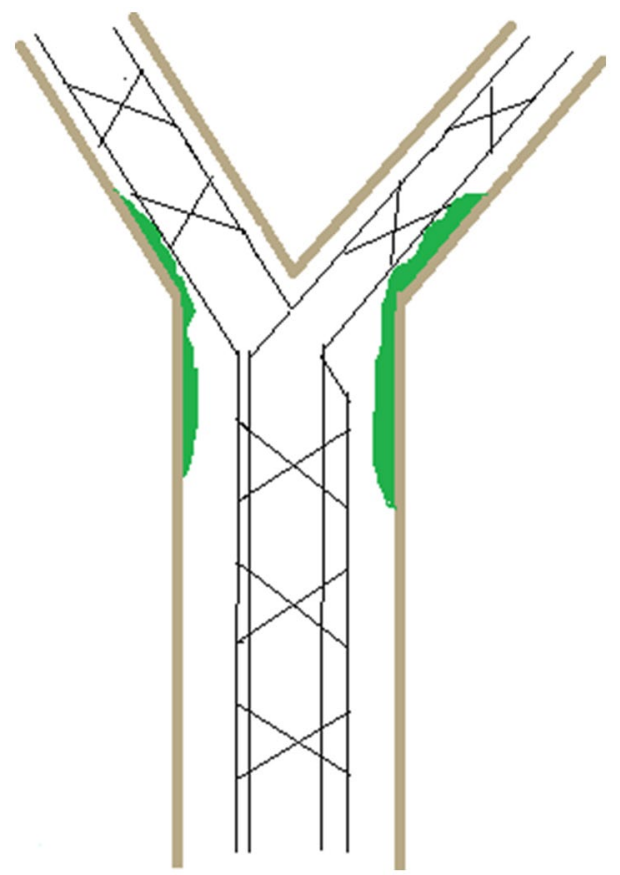

Fig. 1 Illustration of stent-in-stent (SIS) stenting

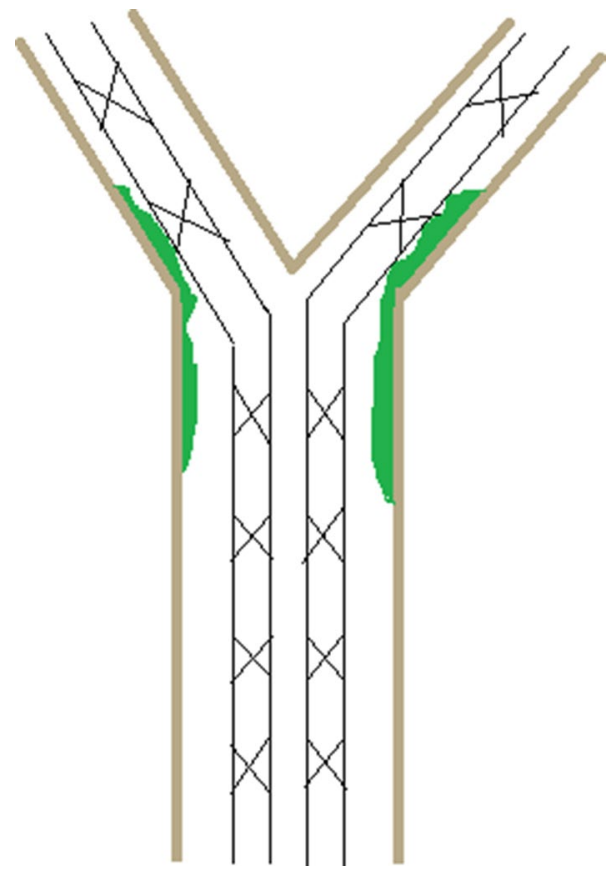

Fig. 2 Illustration of side-by-side (SBS) stenting

reported that assistance by trainees did not impact rates of technical success or adverse events during ERCP [10].

The strengths of the study include the enrollment of a relatively large number of patients for what is a relatively uncommon condition, well-defined outcomes, and thorough long-term follow-up. The authors are to be congratulated as this is a difficult patient population to enroll into prospective protocols, due to the rarity of the disease and short life expectancy. Importantly, outcomes of patients followed until death are provided, a significant contribution of this paper. Furthermore, four study centers participated, adding to the external validity of the findings.

Nevertheless, the study suffers from the usual limitations of a retrospective design; most notably, selection bias. It provides some reassurance that the stent placement method was carried out chronologically and consistently during each study period (SIS 2010-2012 and SBS 2013-2016) rather than according to the endoscopists' preferences. Furthermore, other confounding variables (performance of biliary sphincterotomy, trans-papillary vs supra-papillary placement, trainee involvement) could have impacted the outcome measures. Other potential issues, often common in endoscopic research, include temporal technique refinement and device improvement and availability (e.g., Niti-S ${ }^{\mathrm{TM}}$ large cell D-type stents are not available in many places around the world including the USA). Lastly, findings at expert centers may not be generalizable to lower-volume hospitals.

In everyday practice, unresectable hilar biliary malignancy requires an individualized approach based on the endoscopist's skill set, the types of stents available, and the overall prognosis of the patient. A fundamental principle of this procedure is to maximize liver drainage (arguably requiring bilateral stenting) by stenting the maximum healthy liver volume. Considering the high degree of technical difficulty of ERCP in this patient population, referral to high-volume centers is recommended. Ishigaki, and the current literature, support endoscopic placement of uncovered metal stents using either SBS or SIS depending on operator's preference and stent type availability. Importantly, this study highlights that even in the hands of highly experienced endoscopists, recurrent biliary obstruction following stenting is rather common among patients with malignant hilar obstruction. Therefore, future directions in research should be aimed at considerations of drug-eluting stents, reintervention with endoscopic ultrasound-guided biliary drainage, and local ablative techniques.

As palliative therapy continues to evolve with longer survival times and higher quality-of-life, the ability to perform endoscopic reintervention is essential to achieve satisfactory outcomes. The authors acknowledge, though, that due to the uncommon occurrence of cholangiocarcinoma and the overall poor prognosis of this deadly cancer, studies of this nature and size can be difficult to accomplish. As such, Ishigaki's group presented a well-designed retrospective study with direct practical implications. At present, SBS and SIS should be considered equivalent with the choice between the two techniques based on local expertise and device availability. 
Funding No funding was acquired.

\section{Compliance with Ethical Standards}

Conflict of interest Author Liu has no conflict of Interest. Author Yang is a consultant for Boston Scientific, Lumendi, and Steris. Author Draganov is a consultant for Cook, Boston Scientific, Olympus, and Microtech.

\section{References}

1. Abraham NS, Barkun JS, Barkun AN. Palliation of malignant biliary obstruction: a prospective trial examining impact on quality of life. Gastrointest Endosc. 2002;56:835-841.

2. Al-Kawas F, Aslanian H, Baillie J, et al. Percutaneous transhepatic vs. endoscopic retrograde biliary drainage for suspected malignant hilar obstruction: study protocol for a randomized controlled trial. Trials. 2018;19:108

3. Moss AC, Morris E, Leyden J, MacMathuna P. Do the benefits of metal stents justify the costs? A systematic review and meta-analysis of trials comparing endoscopic stents for malignant biliary obstruction. Eur J Gastroenterol Hepatol. 2007;19:1119-1124.

4. Vienne A, Hobeika E, Gouya H, et al. Prediction of drainage effectiveness during endoscopic stenting of malignant hilar strictures: the role of liver volume assessment. Gastrointest Endosc. 2010;72:728-735.
5. Lee TH, Kim TH, Moon JH, et al. Bilateral versus unilateral placement of metal stents for inoperable high-grade malignant hilar biliary strictures: a multicenter, prospective, randomized study (with video). Gastrointest Endosc. 2017;86:817-827.

6. Naitoh I, Hayashi K, Nakazawa T, et al. Side-by-side versus stent-in-stent deployment in bilateral endoscopic metal stenting for malignant hilar biliary obstruction. Dig Dis Sci. 2012;57:3279-3285.

7. Kim KM, Lee KH, Chung YH, et al. A comparison of bilateral stenting methods for malignant hilar biliary obstruction. Hepatogastroenterology. 2012;59:341-346.

8. Ishigaki K, Hamada T, Nakai Y et al. Retrospective comparative study of side-by-side and stent-in-stent metal stent placmenent for hilar malignant biliary obstruction. Dig Dis Sci. (Epub ahead of print). https://doi.org/10.1007/s10620-020-06155-z.

9. Shin J, Park JS, Jeong S, Lee DH. Comparison of the clinical outcomes of suprapapillary and transpapillary stent insertion in unresectable cholangiocarcinoma with biliary obstruction Dig Dis Sci. 2019.

10. Voiosu T, Boskoski I, Voiosu AM, et al. Impact of trainee involvement on the outcome of ERCP procedures: results of a prospective multicenter observational trial. Endoscopy. 2020;52:115-122.

Publisher's Note Springer Nature remains neutral with regard to jurisdictional claims in published maps and institutional affiliations. 